\title{
Análise da Utilização dos Novos Inibidores da Trombina na Prática Médica
}

\author{
Carolina Blaya, Clarissa Prati, Cristiano Boneti, Daniela Rezende Bonamigo, \\ Rita Carolina Pozzer Krumenauer, Guido Aranha Rosito \\ Porto Alegre, RS
}

A formação da trombina, evento final da cascata da coagulação (fig. 1), parece estar implicada na formação de trombos oclusivos nas coronárias (trombogênese), originando infarto agudo do miocárdio (IAM), angina instável e reoclusões após angioplastia coronária (AC).

A trombina é um polipeptídio plasmático, com peso molecular de 33700UI, que vem sendo relacionado às seguintes funções: catalisar a conversão do fibrinogênio em fibrina; ativar o fator XIII, responsável pela ligação cruzada da fibrina, e estabilização do trombo; estimular a agregação plaquetária, através de receptores acoplados à proteína $\mathrm{G}$; induzir a produção endotelial de fatores pró-trombóticos; potencializar a proliferação de musculatura lisa nos sítios lesados ${ }^{1-4}$

Por ser a trombina considerada o mediador central da trombogênese (hipótese da trombina) acredita-se que a inibição de sua atividade e/ou de seu receptor deva interromper a formação do trombo e diminuir a lesão vascular.

A terapia antitrombótica atual, baseada no uso do ácido-acetilsalicílico (um antiplaquetário) e heparina (inibidor indireto da trombina), tem limitações. A heparina apresenta diversas desvantagens: depende dos níveis séricos de antitrombina III; é uma mistura heterogênea de moléculas, tendo acentuada variabilidade em farmacocinética e farmacodinâmica; é inibida pelo fator plaquetário IV; e, o mais importante, inibe apenas a trombina próxima ao coágulo e não aquela ligada ao trombo, mais relacionada ao processo de reestenose ${ }^{1,5-8}$.

Devido a essas limitações, buscaram-se novas terapias antitrombóticas, estando, entre elas, os inibidores diretos da trombina, que apresentam vantagens teóricas, sendo objeto de vários estudos clínicos.

A hirudina é o protótipo do grupo dos inibidores específicos da trombina, constituído também por hirulog, hirugen e o argatroban ${ }^{9}$.

A hirudina provém da glândula salivar da sanguessuga européia hiruda medicinalis ${ }^{10}$. Para seu uso clínico, a hirudina é produzida por técnicas de DNA recombinante.

Faculdade Federal de Ciências Médicas de Porto Alegre

Correspondência: Guido Aranha Rosito - FFFCMPA - Depto de Farmacologia e

Toxicologia - Rua Sarmento Leite, 245 - $3^{\circ}$ - 90050-170 - Porto Alegre, RS

Recebido para publicação em 24/9/97

Aceito em 26/5/98

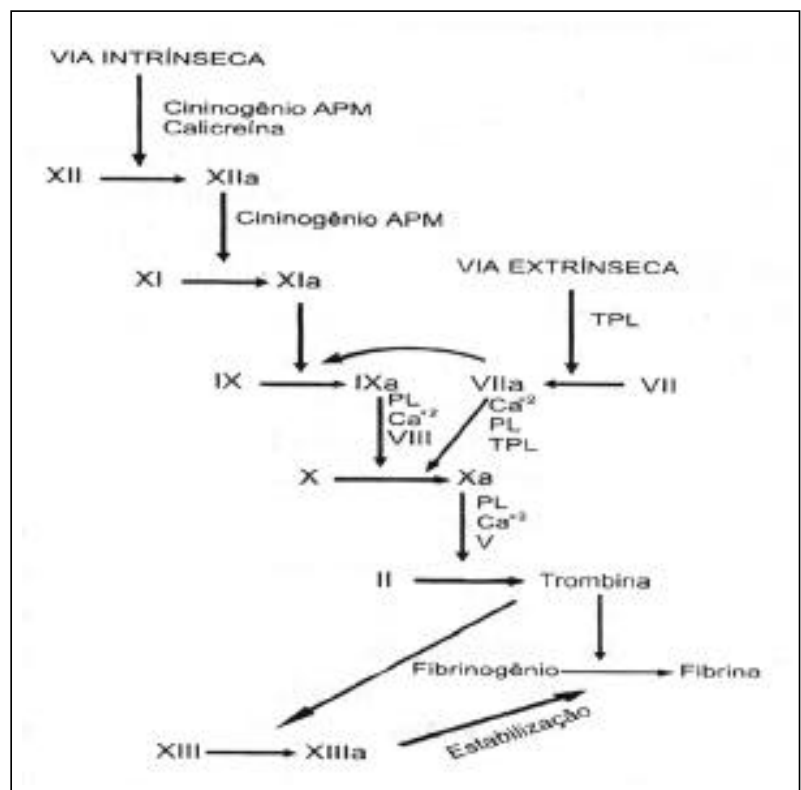

Fig. 1 - Cascata da coagulação - vias intrínseca e extrínseca. TPL- tromboplastina tecidual; APM- alto peso molecular; PL- fosfolipídeos.

Estruturalmente é idêntica à hirudina natural, exceto pela falta de um grupo sulfato na tirosina $63^{1,11}$. Seu mecanismo de ação consiste na ligação a dois sítios da trombina: extremidade C-terminal liga-se ao sítio de reconhecimento do substrato, que seria o local de reconhecimento do fibrinogênio; já a sua extremidade $\mathrm{N}$-terminal liga-se ao sítio catalítico, inibindo a reação auto-catalítica ${ }^{1}$ (fig. 2). Dessa forma, a hirudina não só inibe a conversão do fibrinogênio em fibrina pela trombina, como também, previne a ativação do receptor da trombina ${ }^{2}$. A ligação não é covalente, mas, por possuir uma velocidade de dissociação extremamente lenta, a hirudina é considerada um inibidor essencialmente irreversível ${ }^{1}$.

A hirudina possui vantagens teóricas e práticas sobre a heparina, como inibição da ligação da trombina ao trombo (a heparina não faz esta inibição, pois o sítio de ligação para o complexo heparina-antitrombina III não está acessível, quando a trombina está ligada ao trombo) ${ }^{1}$ (fig. 3). Além disso, a hirudina não necessita de cofator, não é inibida por plaquetas ativadas e causa uma anticoagulação mais estável que a heparina. A hirudina inibe depósitos de fibrina em doses menores do que as necessárias para inibir a deposi- 


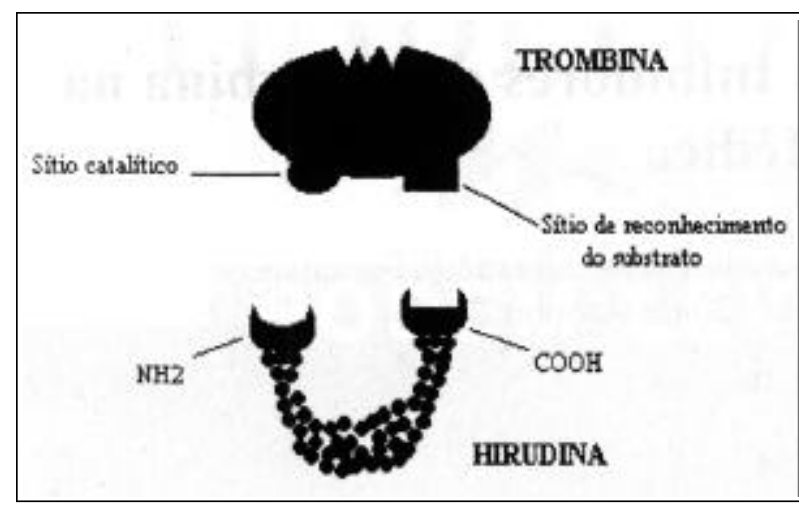

Fig. 2 - Sítios de ligação da trombina para a hirudina. Adaptado de Cannon e Braunwald ${ }^{1}$.

ção de plaquetas ${ }^{12}$. Além disso, enquanto a heparina prolonga o tempo de coagulação ativado de forma definida ${ }^{13}$, a hirudina o faz de forma dose-independente ${ }^{6}$. A heparina pode ser antagonizada pela protamina, enquanto a hirudina não possui antagonistas específicos, até o momento. Entretanto, alguns derivados da protombrina podem neutralizar a hirudina e outros antagonistas da trombina ${ }^{10}$.

O pico plasmático varia entre 3 a $4 \mathrm{~h}$ para $0,1 \mathrm{mg} / \mathrm{kg}$. $\mathrm{O}$ pico de efeito da hirudina sobre o tempo parcial de trombina (TTP) é de 2 a $3 h^{1,14}$. Depois do término da infusão do fármaco, o TTP volta ao normal em 18 a 24h. O volume de distribuição é de $0,231 / \mathrm{kg}^{14}$. O complexo hirudina-trombina circula no sangue entre $4 \mathrm{a} 6 \mathrm{~h}$, sendo eliminado pelo sistema reticuloendotelial. Já a hirudina livre é depurada pelo rim ${ }^{1}$.

O hirulog é um peptídeo sintético composto por 20 aminoácidos. Assim como a hirudina, possui um grupo $\mathrm{COOH}$ terminal, que se liga ao sítio de reconhecimento do substrato da trombina e um grupo $\mathrm{NH}_{2}$ terminal, capaz de bloquear o sítio de atividade catalítica. $\mathrm{O}$ hirulog inibe, reversivelmente, a maioria das ações da trombina, incluindo a clivagem de fibrinogênio em fibrina, a ativação de plaquetas e a ativação de fatores V e VIII (responsáveis por uma ação de amplificação geradora de mais trombina) ${ }^{6}$. Esse fármaco tem um tempo de meia-vida de $36 \mathrm{~min}$ e $20 \%$ da dose é eliminada pelo rim $^{8}$.

\section{Ensaios clínicos}

Infarto agudo do miocárdio - A hirudina, como terapia adjunta aos trombolíticos no IAM, foi avaliada em vários estudos clínicos randomizados. Inicialmente, o estudo piloto TIMI-5 randomizou 246 pacientes, que receberam heparina ou hirudina $(0,15,0,30,045$ e $0,6 \mathrm{mg} / \mathrm{kg})$ seguidos de infusão de $0,05 \mathrm{mg} / \mathrm{kg} / \mathrm{h}$ a $0,6 \mathrm{mg} / \mathrm{kg} / \mathrm{h}$, associados a t-PA e ácido-acetilsalicílico. Os resultados obtidos revelaram reperfusões ótimas em todos os grupos em 90min de seguimento; entretanto, após 18 a 36h de terapia, a hirudina obteve melhor grau de reperfusão da artéria relacionada ao infarto. Quanto à reoclusão dessa artéria, sangramento e morte, a hirudina também mostrou-se melhor que a heparina ${ }^{11}$.

Um outro estudo piloto, TIMI-6, avaliou a hirudina como adjunta à estreptoquinase e ao ácido-acetilsalicílico

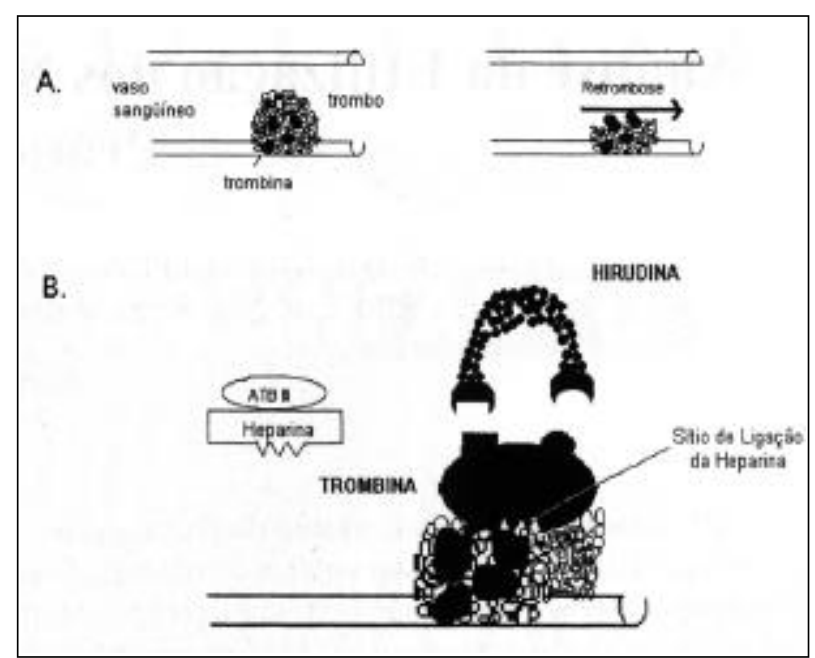

Fig. 3 - Papel da trombina na progressão de trombose. A trombina é incorporada com a fibrina dentro do coágulo. A fibrinólise endógena ou por terapia trombolítica provoca a exposição das moléculas de trombina que podem, assim, induzir retrombose (A). O complexo heparina-antitrombina III (ATB III) não inibe a trombina ligada ao coágulo, pois o sítio de ligação à heparina está encoberto. Já a hirudina é capaz de inibir a trombina ligada ao coágulo. Adaptado de Cannon e Braunwald ${ }^{1}$.

em 193 pacientes. Os pacientes foram randomizados a receberem um tratamento por cinco dias com infusão de $5000 \mathrm{U}$ de heparina em bolo seguida por 5000U/h ajustado pelo TTP (65-90s), ou uma infusão constante de uma das três seguintes doses: bolo de $0,15,0,3$, ou $0,6 \mathrm{mg} / \mathrm{kg}$ seguidos de mais $0,05,0,1$ ou $0,2 \mathrm{mg} / \mathrm{kg} / \mathrm{h}$. Os resultados revelaram uma incidência de hemorragias semelhante nos diferentes grupos. A incidência de morte, infarto não fatal, insuficiência cardíaca congestiva (ICC) ou choque cardiogênico foi maior no grupo da dose baixa de hirudina do que nas doses mais altas ${ }^{15}$.

A partir dos resultados favoráveis obtidos nos estudos de nível II, iniciou-se estudos de nível III.

O ensaio clínico TIMI-9A randomizou 757 pacientes com diagnóstico de IAM para receberem heparina em doses ajustadas, conforme o peso, em bolo de 5000U seguido de infusão de $1000 \mathrm{U} / \mathrm{h}$ para paciente, com menos de $80 \mathrm{~kg}$ ou $1300 \mathrm{U} / \mathrm{h}$ para pacientes com peso $\geq 80 \mathrm{~kg}$, ou hirudina em bolo de $0,6 \mathrm{mg} / \mathrm{kg}$ seguido de $0,2 \mathrm{mg} / \mathrm{kg} / \mathrm{h}$ durante $96 \mathrm{~h}$. Esse estudo foi suspenso devido ao aparecimento de hemorragias acima do esperado em ambos grupos ${ }^{16}$.

O ensaio clínico GUSTO-IIa (Global use of strategies to open occluded arteries) randomizou 256 pacientes com angina e alterações no eletrocardiogama para receberem hirudina ou heparina nas mesmas doses do TIMI-9A ${ }^{17}$.

Entre os estudos TIMI-9A e GUSTO-IIa, encontrou-se uma incidência semelhante de hemorragia endocraniana associada a um nível de TTP mais elevado ${ }^{18}$; contudo, a incidência de hemorragias não intracranianas foi maior no grupo da hirudina, de acordo com o estudo TIMI-9A. Devido a isso, ambos estudos - TIMI-9A e GUSTO-IIa - sugeriram para os próximos estudos uma redução na dose de hirudina para um bolo de $0,1 \mathrm{mg} / \mathrm{kg}$ seguido de infusão de $0,1 \mathrm{mg} / \mathrm{kg} / \mathrm{h}$. Além disso, devido à relação do TTP com os efeitos hemorrágicos, as infusões de hirudina e heparina 


\begin{tabular}{|c|c|c|c|c|c|}
\hline Doença & Estudo & $\mathrm{N}^{\mathrm{o}}$ de pacientes & Fármaco & $\begin{array}{l}\text { Dose } \\
(\mathrm{mg} / \mathrm{kg})\end{array}$ & Conclusões \\
\hline Angina instável & TIMI $7^{6}$ & 410 & $\begin{array}{l}\text { hirulog + ácido- } \\
\text { acetilsalicílico }\end{array}$ & $\begin{array}{l}\text { infusão } \\
0,2-1\end{array}$ & $\begin{array}{l}\text { Doses mais elevadas reduziram } \\
\text { a mortalidade em relação às } \\
\text { doses mais baixas de } 6,8 \% *\end{array}$ \\
\hline IAM & TIMI $9 b^{7}$ & 3002 & $\begin{array}{l}\text { hirudina }+ \text { es- } \\
\text { treptoquinase } \\
\text { ou }+\mathrm{t}-\mathrm{PA}\end{array}$ & $\begin{array}{l}\text { bolo } 0,1 \\
\text { infusão } 0.1\end{array}$ & Eficácia equivalente a heparina \\
\hline IAM & TIMI $5^{11}$ & 246 & $\begin{array}{l}\text { hirudina +ácido- } \\
\text { acetilsalicílico } \\
\text { + t-PA }\end{array}$ & $\begin{array}{l}\text { bolo } 0,15-0,6 \\
\text { infusão } \\
00,5-0,6\end{array}$ & $\begin{array}{l}\text { Aumento da reperfusão e } \\
\text { redução de reinfarto e } \\
\text { morte de } 9,9 \% *\end{array}$ \\
\hline IAM & TIMI $6^{15}$ & 193 & $\begin{array}{l}\text { hirudina +ácido- } \\
\text { acetilsalicílico } \\
\text { + estreptoquinase }\end{array}$ & $\begin{array}{l}\text { bolo } \\
0,15-0,6 \\
\text { infusão } \\
0,05-0,2\end{array}$ & $\begin{array}{l}\text { Incidência de morte, IAM, ICC } \\
\text { ou choque cardiogênico maior } \\
\text { nas doses mais baixas do que } \\
\text { nas mais altas de } 11,2 \% *\end{array}$ \\
\hline IAM & TIMI $9 \mathrm{a}^{16}$ & 757 & hirudina & $\begin{array}{l}\text { bolo } 0,6 \\
\text { infusão } 0,2\end{array}$ & $\begin{array}{l}\text { Suspenso por alta incidência } \\
\text { de hemorragias intracranianas }\end{array}$ \\
\hline IAM & GUSTO IIa $^{17}$ & 256 & hirudina & $\begin{array}{l}\text { bolo } 0,6 \\
\text { infusão } 0,2\end{array}$ & $\begin{array}{l}\text { Suspenso por alta incidência } \\
\text { de hemorragias intracranianas }\end{array}$ \\
\hline IAM & GUSTO IIb ${ }^{19}$ & 12142 & hirudina & $\begin{array}{l}\text { bolo } 0,1 \\
\text { infusão } 0,1\end{array}$ & $\begin{array}{l}\text { Redução na incidência de } \\
\text { morte e infarto não fatal de } 0,9 *\end{array}$ \\
\hline
\end{tabular}

passaram a ser monitoradas por um TTP entre 55 e $85 \mathrm{~s}^{16,17}$. O estudo TIMI-9B randomizou 3002 pacientes com IAM, tratados com ácido-acetilsalicílico e t-PA ou estreptoquinase, para receberem heparina em bolo de $5000 \mathrm{U}$ seguida de $1000 \mathrm{U} / \mathrm{h}$ independente do peso do paciente, ou hirudina em bolo de $0,1 \mathrm{mg} / \mathrm{kg}$ (sem exceder $15 \mathrm{mg}$ ), seguido por infusão contínua de $0,1 \mathrm{mg} / \mathrm{kg} / \mathrm{h}$ (sem exceder $15 \mathrm{mg} / \mathrm{h}$ ). $\mathrm{O}$ ajuste de ambas as doses foi feito pelo TTP (mantido entre 55 e $85 \mathrm{~s}$ ). A hirudina e a heparina mostraram-se igualmente efetivas no que se refere ao efeito antitrombótico e às seguintes complicações: IAM não fatal, ICC ou choque cardiogênico e hemorragias maiores. Considerando sua eficácia semelhante, a hirudina seria uma alternativa atraente para pacientes com história de hipersensibilidade ou desenvolvimento de trombocitopenia induzida por heparina ${ }^{7}$.

O GUSTO-IIb randomizou 12142 pacientes com síndromes coronárias agudas a receberem heparina ou hirudina nas mesmas doses do estudo acima, sendo as doses de infusão mantidas em nível de TTP entre 60 e 90s. Divergindo do TIMI-9B, houve uma redução de $11 \%$ na incidência de morte e de reinfarto não fatal em 30 dias em comparação com a heparina. Além disso não se evidenciou diferença estatisticamente significativa na incidência de sangramentos graves, mas a hirudina estava associada a uma maior incidência de sangramentos moderados ${ }^{19}$.

Num estudo randomizado duplo-cego, duas doses de hirulog foram comparadas com a heparina em 68 pacientes com IAM, tratados com estreptoquinase e ácido-acetilsalicílico. Os pacientes foram divididos em três grupos: $01^{\circ}$ grupo recebeu placebo em bolo e $0,5 \mathrm{mg} / \mathrm{kg} / \mathrm{h}$ nas primeiras $12 \mathrm{~h}$, seguido por $0,1 \mathrm{mg} / \mathrm{kg} / \mathrm{h}$ por $4-6$ dias; o $2^{\circ}$ recebeu placebo em bolo e $1 \mathrm{mg} / \mathrm{kg} / \mathrm{h}$ nas primeiras $12 \mathrm{~h}$, seguido por placebo por 4-6 dias; e o $3^{\circ}$ recebeu $5000 \mathrm{U}$ de heparina em bolo e 1000U/h de heparina nas primeiras $12 \mathrm{~h}$. Os resultados demonstraram que o hirulog promove a revascularização da artéria responsável pelo infarto melhor que a heparina. Além disso, doses elevadas de hirulog não são mais eficazes que as doses baixas. Entretanto esses achados devem ser confirmados com estudos maiores ${ }^{20}$ (tab. I).

O ensaio AMI randomizou 910 pacientes com IAM para serem tratados com doses baixas ou médias de argratrobam ou placebo, como terapia adjuvante à estreptoquinase. A terapia com argratrobam demonstrou-se segura, porém não houve diferença estatística entre os grupos em relação aos desfechos morte, choque, ICC ou recorrência do IAM em 30 dias $^{21}$.

Angina instável - Em um estudo randomizado, a hirudina foi comparada com a heparina no tratamento da angina instável. Os 166 participantes com evidência angiográfica de trombose coronária receberam uma das duas doses de heparina (a $1^{\mathrm{a}}$ tendo como alvo um TTP de 65 a 90 s e a $2^{\text {a }}$, de 90 a 110 s) ou uma das quatro doses de hirudina (variando entre $0,15 \mathrm{mg} / \mathrm{kg}$ em bolo seguido de infusão constante de $0,05 \mathrm{mg} / \mathrm{kg} / \mathrm{h}$ até $0,6 \mathrm{mg} / \mathrm{kg}$ em bolo mais infusão de $0,3 \mathrm{mg} / \mathrm{kg}$ ) por cinco dias. A hirudina recombinante mostrou-se superior em aumentar o calibre da secção transversa do vaso obstruído; causou elevação mais consistente e estável no TTP; e promoveu um melhor efeito antitrombótico in vivo do que a heparina, apesar de os resultados in vitro serem semelhantes ${ }^{22}$.

A dose ideal de hirulog no tratamento da angina instável foi investigado no TIMI-7, um ensaio clínico randomizado duplo-cego, envolvendo 410 pacientes, que receberam, juntamente com $325 \mathrm{mg} /$ dia de ácido-acetilsalicílico, uma das quatro doses de hirulog em infusão contínua por 
72h: 0,02, 0,25, 0,5 e 1mg/kg/h. A eficácia - avaliada conforme o desenvolvimento de IAM, morte, isquemia recorrente ao repouso e rápida piora clínica durante o tratamento - não foi diferente entre as quatro doses. Com a dose menor, ocorreu IAM não fatal ou morte nos seis meses seguintes à alta hospitalar em $10 \%$ dos pacientes, enquanto que com as três maiores doses essa taxa foi de 3,2\%. Os resultados do TIMI7 também mostram que o hirulog, quando associado ao ácido-acetilsalicílico, parece ser bem tolerado e promove um TTP estável sem reajuste de dose ${ }^{6}$ (tab. I).

Angioplastia coronária - Um estudo foi realizado com 291 pacientes submetidos à AC eletiva, com a intenção de determinar a viabilidade de uso e a dose ideal de hirulog, como único anticoagulante neste procedimento. Os pacientes foram divididos em cinco grupos que receberam as seguintes doses de hirulog: 1) $0,15 \mathrm{mg} / \mathrm{kg}$ em bolo e $0,6 \mathrm{mg} / \mathrm{kg} /$ $\mathrm{h}$ por $4 \mathrm{~h} ; 2$ ) $0,25 \mathrm{mg} / \mathrm{kg}$ em bolo e $1 \mathrm{mg} / \mathrm{kg} / \mathrm{h}$ por $4 \mathrm{~h} ; 3$ ) $0,35 \mathrm{mg} / \mathrm{kg}$ e $1,4 \mathrm{mg} / \mathrm{kg} / \mathrm{h}$ por $4 \mathrm{~h} ; 4) 0,45 \mathrm{mg} / \mathrm{kg}$ e $1,8 \mathrm{mg} / \mathrm{kg} / \mathrm{h}$ por $4 \mathrm{~h} ; 5)$,, $55 \mathrm{mg} / \mathrm{kg}$ e $2,2 \mathrm{mg} / \mathrm{kg} / \mathrm{h}$ por $4 \mathrm{~h}$. Foi monitorado o TTP e o ACT dos pacientes. Os resultados desse estudo demonstraram que o fechamento abrupto do vaso, após a cirurgia, ocorreu em 11,3\% dos paciente dos grupos 1,2,3e, em apenas, 3,9\% dos pacientes dos grupos 4 e 5 . Não houve complicações hemorrágicas significativas. Esse estudo foi o primeiro que documentou a possibilidade do uso de um coagulante, que não a heparina, na AC. Nele, o hirulog foi associado com um efeito dose-dependente, mínimas complicações hemorrágicas e baixa taxa de fechamento abrupta de vaso nas doses de $1,8-2,2 \mathrm{mg} / \mathrm{kg}^{26}$.

Os efeitos do hirulog em pacientes submetidos a AC, também, têm sido comparados por alguns autores com os da heparina. Há um estudo duplo-cego, randomizado, realizado com 4098 indivíduos que sofreram AC devido a angina instável e pós-infarto. Os pacientes receberam, imediatamente antes do procedimento, uma dose em bolo de $1,0 \mathrm{mg} /$ $\mathrm{kg}$ de hirulog, seguida de $4 \mathrm{~h}$ de infusão de $2,5 \mathrm{mg} / \mathrm{kg} / \mathrm{h}$ e mais $14 \mathrm{a} 20 \mathrm{~h}$ de $0,2 \mathrm{mg} / \mathrm{kg} / \mathrm{h}$ da droga. O grupo controle foi tratado com heparina em altas doses, regime constituído de dose em bolo de $175 \mathrm{U} / \mathrm{kg}$ e infusão por $18-24 \mathrm{~h} \mathrm{de} 15 \mathrm{U} / \mathrm{kg} / \mathrm{h}$. Os resultados mostraram, que hirulog não reduziu significativamente a incidência de morte no período hospitalar, mas resultou em diminuição de sangramentos. Com um seguimento de seis meses, o número de mortes, IAM e revascularizações não foi diferente entre os dois grupos. Concluiu-se que hirulog é tão efetivo quanto altas doses de heparina na prevenção de complicações isquêmicas na AC para angina instável, além de diminuir a incidência de sangramentos. As complicações isquêmicas agudas foram reduzidas nos pacientes com angina pós-infarto, porém esse resultado não se confirmou a longo prazo ${ }^{27}$.

Os efeitos adversos do tratamento com hirulog foram analisados em outro estudo, com 4312 pacientes. A terapia com hirulog ocasionou taxas menores de hematúria, hemorragia grave e hematêmese, e equivalentes de morte, IAM e efeitos adversos em outros órgãos, quando comparada com heparina ${ }^{28}$.
O uso de hirudina na angioplastia não teve benefícios clínicos em seguimento a longo prazo, como foi descrito em um estudo randomizado com 1141 pacientes. Nesse estudo, os indivíduos receberam: 1) dose em bolo de $10.000 \mathrm{U}$ de heparina, seguida de infusão de heparina por 24h e administração subcutânea (sc) de solução placebo duas vezes por dia, durante três dias; 2) dose em bolo de $40 \mathrm{mg}$ de hirudina, seguida de infusão da droga por $24 \mathrm{~h}$ e o mesmo regime de placebo;3) mesmo esquema de hirudina mais 40mg da droga subcutâneo ao invés de placebo. Após sete meses, não houve intercorrências clínicas em nenhum dos três grupos, porém a administração de hirudina sc (grupo 3) esteve associada com significativa redução de eventos cardíacos agudos ${ }^{29}$.

Um outro estudo avaliou o uso de hirudina em comparação à heparina em pacientes com angina estável submetidos a angioplastia com balão. Os resultados obtidos indicaram que ocorreram mais sangramentos com a hirudina, apesar do TTP dos pacientes tratados com hirudina terem alcançado a anticoagulação almejada com maior frequiência e de forma mais constante. Quanto ao resultado do procedimento, o uso de hirudina reduziu as reoclusões e obteve melhor fluxo em relação à heparina ${ }^{30}$.

Trombose venosa profunda-Um estudo randomizado multicêntrico duplo-cego avaliou três doses de hirudina (10, 15 ou $20 \mathrm{mg} \mathrm{sc} 2 \mathrm{x} / \mathrm{dia}$ ) comparada com a heparina ( $5000 \mathrm{U} \mathrm{sc}$ $3 \mathrm{x} /$ dia ) em 1119 pacientes submetidos a cirurgia eletiva de quadril. Todas as doses de hirudina demonstraram-se mais efetivas que a heparina em reduzir trombose venosa proximal. Apesar de as doses de 15 e $20 \mathrm{mg}$ demonstrarem um maior benefício clínico, não foi verificada uma relação dose-dependente. Os quatro grupos não demonstraram diferença estatisticamente significativa, quanto ao sangramento. Conclui-se que a hirudina é mais eficaz que a heparina em prevenir tromboembolismo, após cirurgia eletiva de quadril, sendo que a dose fixa de 15 ou $20 \mathrm{mg}$ de hirudina sc, duas vezes ao dia, iniciada no período pré-operatório e estendendo-se por nove dias é a profilaxia mais segura e eficaz ${ }^{23}$.

Outro estudo randomizado duplo-cego avaliou as mesmas doses de hirudina, comparada com a heparina, em 148 pacientes submetidos a cirurgia eletiva de quadril. A hirudina demonstrou redução significativa das complicações tromboembólicas. As três doses de hirudina demonstraram-se seguras, confirmando os achados do estudo anteriormente descrito, induzindo anticoagulação sistêmica mais pronunciada, que a heparina sem interferir com sangramentos ou necessidade de transfusão ${ }^{24}$.

Um estudo multicêntrico randomizou 2079 pacientes submetidos a artroplastia total de quadril em dois grupos, onde um recebeu $15 \mathrm{mg} \mathrm{sc}$ de hirudina duas vezes ao dia e o outro recebeu 40mg sc de heparina de baixo peso molecular uma vez ao dia. Ambos os tratamentos iniciaram-se no período pré-operatório e estenderam-se por oito a 12 dias. Houve uma redução significativa de $40 \%$ na incidência de trombose venosa profunda proximal no grupo que recebeu hirudina em relação ao grupo que recebeu heparina de baixo 
peso molecular. Além disso, encontrou-se também uma diminuição na incidência total de TVP de $28 \%$ no grupo que recebeu hirudina ${ }^{25}$.

\section{Conclusão}

As evidências embasadas em grandes estudos clínicos randomizados apóiam o uso da hirudina no IAM. Analisados em conjunto, GUSTO-IIb e TIMI-9B mostraram uma redução de $13 \%$ na incidência de reinfarto por 30 dias, quando comparados à heparina. Entretanto não foi constatada alteração na mortalidade total ${ }^{31}$. Na angina instável, o seu uso parece promissor, carecendo de estudos clínicos com desfechos cardiovasculares como IAM e morte súbita. Em relação à angioplastia, os resultados são controversos. Na prevenção de TVP, após cirurgia de quadril, houve marcada superioridade da hirudina, quando comparada à heparina de baixo peso molecular com um desfecho não-clínico. Esta vantagem potencial necessita ser confirmada em estudos maiores com desfechos clínicos importantes, como incidência de embolia pulmonar.

Apesar do uso da hirudina ser controverso em algumas situações, uma indicação já bem definida é em pacientes com hipersensibilidade ou trombocitopenia induzida pela heparina $^{32}$.

Novos ensaios clínicos, envolvendo um maior número de pacientes e diferentes esquemas de administração, são necessários para aprimorar os conhecimentos a respeito desses fármacos e para que se possa assegurar o seu emprego, como terapia segura e mais eficaz que as atuais.

\section{Referências}

1. Cannon CP, Braunwald E - Hirudin: initial resuls in acute myocardial infarction, unstable angina and angioplasty. J Am Coll Cardiol 1995; 20: 30S-7S.

2. Baykal D, Schmedtje JF, Runge MS - Role of the thrombin receptor in restenosis and atherosclerosis. Am J Cardiol 1995; 75: 82B-7B.

3. Brass LF - Issues in the development of thrombin receptor antagonists. Thromb Haemost 1995; 74: 499-505

4. Harker LA, Hanson SR, Runge MS - Thrombin hypothesis of thrombus generation and vascular lesion formation. Am J Cardiol 1995; 75: 12B-17B.

5. Ginsberg JS, Nurmohamed MT, Gent M et al - Effecs on thrombin generation of single injections of HirulogTM in patients with calf vein thrombosis. Thromb Haemost 1994; 72: 523-5.

6. Fuhcs J, Cannon CP and the TIMI 7 Investigators. Hirulog in the treatment of unstable angina - Resuls of the Thrombin Inhibition in Myocardial Ischemia (TIMI) 7 Trial. Circulation 1995; 92: 727-33

7. Antman EM for the TIMI 9B Investigators - Hirudin in acute myocardial infarction - Thrombolysis and Thrombin Inhibition in Myocardial Infarction (TIMI) 9B Trial. Circulation 1996; 94: 911-21.

8. Hamelink JK, Tang DB, Barr C F et al - Inhibition of platelet deposition by combined hirulog and aspirin in a rat carotid endarterectomy model. J Vasc Surg 1995; $21: 492-8$

9. Gersh BJ, Opie LH - Antithrombotic agents: platelet inhibitors, anticoagulants, and fibrinolytics. In: Opie LH - Drugs for the Heart $4^{\text {th }}$. Ed. Philadelphia: W B Saunders, 1995: 262

10. Fisher BE, Scholakat U, Mitterer A et al - Rational design, recombinant preparation, and in vitro and in vivo characterization of human prothrombin-derived hirudin antagonists. J Biol Chem 1996; 271: 23737-42.

11. Scharfstein JS, Abendschein DR, Cannon CP et al - A pilot trial of recombinant desulfatohirudin compared with heparin in conjunction with tissue-type plasminogen activator and aspirin for acute myocardial infarction: results of the Thrombolysis in Myocardial Infarction (TIMI) 5 trial. J Am Coll Cardiol 1994; 23: 993-1003

12. Jackson MR et al - Antithrombotic effecs of hirulog in rat carotid endarterectomy model. J Surg Res 1996; 60: 15-22.

13. Carteaux JP, Gast A, Tshopp TB, Roux S - Activated clotting time as an appropriate test to compare heparin and direct thrombin inhibitors such as hirudin or ro 46-6240 in experimental arterial thrombosis. Circulation 1995; 91: 1568-74.

14. Thürmann $P$, Harder S, Kirchmaier CM - Influence of piroxican coadministration on pharmacodynamic parameters and the plasma concentration/effect relationship of recombinant hirudin (CGP 39393). Eur J Clin Pharmacol 1995; 48: 241-6.

15. Lee LV for the TIMI 6 Investigators. Initial experience with hirudin and streptokinase in acute myocardial infarction: results of the TIMI 6 trial. Am J Cardiol 1995; 75: 7-13.

16. Antman E - Hirudin in acute myocardial infarction. Safety report from the Thrombolysis and Thrombin Inhibition in Myocardial Infarction (TIMI) 9 A Trial. Circulation 1994; 90: 2147-52.
17. The Global Use of Strategies to Open Occluded Coronary Arteries (GUSTO) IIa Investigators. Randomized Trial of intravenous heparin versus recombinant hirudin for acute coronary syndromes. Circulation 1994; 90: 1631-7.

18. Cannon CP et al - Usefulness of APTT to predict bledding for hirudin (and heparin). Circulation 1994; 90(suppl I) (Pt 2): I-563.

19. The Global Use of Strategies to Open Occluded Coronary Arteries (GUSTO) IIb Investigators. A comparision of recombinant hirudin with heparin for the treatment of acute coronary syndromes. N Engl J Med 1996; 335: 775-782.

20. Théroux P, Pérez-Villa F, Waters D, Lespérance J, Shabani F, Bonan R - Randomized double-blind comparision of two doses of hirulog with heparin as adjunctive therapy to streptokinase to promote early patency of the infarct-related artery in acute myocardial infarction. Circulation 1995; 91: 2132- 8.

21. Cody RJ - Results from late breaking clinical trials sessions at ACC'97. J Am Coll Cardiol 1997; 30: 1-7.

22. Topol EJ, Fuster V, Harrington RA et al - Recombinant hirudin for unstable angina pectoris - a multicenter, randomized angiographic trial. Circulation 1994; 89: 1557- 66.

23. Eriksson BI, Ekman S, Kälebo P, Zachrisson B, Bach D, Close P - Prevention of deep-vein thrombosis after total hip replacement: direct thrombin inhibition with recombinant hirudin, CGP 39393. The Lancet 1996; 347: 635-9.

24. Cofrancesco E, Cortelaro M, Leonardi P, Corradi A, Ravasi F, Bertocchi F - Markers of hemostatic system activation during thromboprophylaxis recombinant hirudin in total hip replacement. Thromb Haemost 1996; 75: 407-11.

25. Eriksson BI, Wille-Jorgensen P, Kälebo P et al - A comparison of recombinant hirudin with a low-molecular-weight heparin to prevent thromboembolic complications after total hip replacement. The New England Journal of Medicine 1997; 337: 1329-35.

26. Topol EJ, Bonan R, Jewitt Det al - Use of antithrombin, hirulog, in place of heparin during coronary angioplasty. Circulation, 1993; 87: 1622-9.

27. Bittl JA, Strony J, Brinker JA et al for the Hirulog Angioplasty Study Investigators. Treatment with bivalirudin hirulog as compared with heparin during coronary angioplasty for unstable or postinfarction angina. N Engl J Med, 1995; 333: 764-9.

28. Bittl JA on behalf of the Hirulog Angioplasty Study Investigators - Comparative safety profiles of hirulog and heparin in patients undergoing coronary angioplasty. Am Heart J, 1995; 130: 658-65.

29. Serruys PW, Herrman JRP, Simon R et al for the Helvetica Investigators - A comparison of hirudin with heparin in the prevention of restenosis after coronary angioplasty. N Engl J Med 1995; 333: 757-63.

30. Van Den Bos AA, Deckers JW, Heyndrickx GR et al - Safety and efficacy of recombinant hirudin (CGP 39393 ) versus heparin in patients with stable angina undergoing coronary angioplasty. Circulation 1993; 88: 2058-65.

31. Van Der Werf F - International workshop on antithrombotic therapy for acute coronary syndromes. Circulation 1996; 94: 593.

32. Walenga JM, Fareed $\mathbf{J}$ - Current status on new anticoagulant and antithrombotic drugs and devices. Curr Opin Pulm Med, 1997; 3: 291-302. 